\title{
Computerized Adventitious Respiratory Sounds as Outcome Measures for Respiratory Therapy: A Systematic Review
}

\author{
Alda Marques PhD, Ana Oliveira PT, and Cristina Jácome MSc
}

\begin{abstract}
INTRODUCTION: There is a need to develop simple, noninvasive, and sensitive outcome measures for respiratory therapy. Adventitious respiratory sounds (ie, crackles and wheezes) can be objectively characterized with computerized respiratory sound analysis (CORSA) and have been shown to contribute for diagnosis purposes; however, their potential for use as outcome measures is unknown. Thus, this systematic review synthesizes the evidence on the use of computerized adventitious respiratory sounds as outcome measures. METHODS: The Web of Knowledge, MEDLINE, EMBASE, and SCOPUS databases were searched. Reviewers independently selected studies according to the eligibility criteria. Effect sizes and 95\% CIs were computed. RESULTS: Twelve studies with different designs (observational, $n=3$; quasi-experimental $n=7$; and randomized controlled trial, $n=2$ ) were included. Eight studies were conducted with adults, and 4 studies with children. Most studies explored only one type of adventitious respiratory sound. For wheezes, the occupation rate seemed to be the most promising parameter to be used as an outcome measure, with high/medium effect sizes $(0.62-1.82)$. For crackles, the largest deflection width showed high effect sizes (1.31 and 1.04); however, this was explored in only one study. Crackle number and 2-cycle duration presented conflicting information, with high/poor effect sizes depending on the study. CONCLUSIONS: Specific variables of each adventitious respiratory sound detected and characterized by CORSA showed high effect sizes and, thus, the potential to be used as outcome measures. Further research with robust study designs and larger samples (both of children and adult populations), and following CORSA guidelines is needed to build evidence-based knowledge on this topic. Key words: computerized respiratory sound analysis; respiratory sounds; adventitious respiratory sounds; wheezes; crackles; outcome measure. [Respir Care 2014;59(5):765-776. () 2014 Daedalus Enterprises]
\end{abstract}

\section{Introduction}

Respiratory diseases are a major cause of societal, health, and economic burdens worldwide. ${ }^{1}$ Therefore, in the last

\footnotetext{
Dr Marques, Ms Oliveira, and Ms Jácome are affiliated with the School of Health Sciences, University of Aveiro (ESSUA), Aveiro, Portugal. Dr Marques is affiliated with Unidade de Investigação e Formação sobre Adultos e Idosos (UniFAI), Porto, Portugal. Ms Jácome is affiliated with the Research Centre in Physical Activity, Health and Leisure, Faculty of Sports, University of Porto, Porto, Portugal.
}

This work was funded by Fundação para a Ciência e a Tecnologia, Portugal (Project: Adventitious lung sounds as indicators of severity and recovery of lung pathology and sputum location- PTDC/SAU-BEB/ 101943/2008).

The authors have disclosed no conflicts of interest. decade, significant research efforts have been dedicated to improve early diagnosis and routine monitoring of patients with respiratory diseases to allow timely interventions. However, addressing these issues has been highly challenging since available respiratory measures such as spirometry are dependent on patient motivation and cooperation, and other tests such as blood gas analysis and imaging are not available in all clinical settings and are expensive. ${ }^{2,3}$

\footnotetext{
Correspondence: Alda Marques PhD, School of Health Sciences, University of Aveiro (ESSUA), Agras do Crasto-Campus Universitário de Santiago, Edifício 30, 3810-193 Aveiro, Portugal. E-mail: amarques@ ua.pt.

DOI: $10.4187 /$ respcare. 02765
} 
Computerized respiratory sound analysis (CORSA), which consists of recording patients' respiratory sounds with an electronic device and analyzing them based on specific signal characteristics, is a simple, objective, and noninvasive method to detect and characterize adventitious respiratory sounds (ARSs), ie, crackles (CRs) and wheezes (WHs). ARSs provide crucial information on respiratory dysfunction, ${ }^{4}$ and changes in their characteristics (eg, intensity, duration, timing) might inform the clinical course of respiratory diseases and treatments. ${ }^{5,6}$ Through the use of CORSA, ARSs have been found to be a more sensitive indicator, detecting and characterizing the severity of the respiratory disease before any other measure. ${ }^{7}$ Thus, this approach, through objective data collection and management, generation of permanent records of the measurements made with easy retrievability, and graphic representations, assists with the diagnosis and monitoring of respiratory diseases. ${ }^{8-11}$

Research on this topic has been focusing on the use of CORSA as a diagnostic aid ${ }^{12}$ and the findings reporting its potential to be used as an outcome measure, that is, to monitor respiratory treatments, are widespread in the literature. Thus, this systematic review synthesizes the evidence on the use of computerized ARSs as outcome measures.

\section{Methods}

\section{Search Strategy}

An electronic literature search was performed from December 2012 to January 2013 in Web of Knowledge (19702012), MEDLINE (1948-2012), EMBASE (1974-2012), and SCOPUS (1960-2013) databases. Search terms were based on a combination of the following terms: monitor* OR "computerized analyses" OR "digital auscultation" OR "electronic auscultation" OR "automatic auscultation" OR "acoustic signal processing" AND "added lung sounds" OR "abnormal lung sounds" OR "adventitious lung sounds" OR "adventitious respiratory sounds" OR crackle* OR wheez*. The search terms were limited to titles and abstracts. The reference lists of the selected articles were scanned for other potential eligible studies. Additionally, a weekly update was conducted until June 2013. This systematic review is reported according to Preferred Reporting Items for Systematic Reviews and Meta-Analyses (PRISMA) guidelines. ${ }^{13}$

\section{Eligibility Criteria}

Articles were included in the study if (1) they detected and characterized ARSs with CORSA before and after an intervention in adults or children; (2) were experimental, quasi-experimental, or observational studies; (3) were full papers published as original articles or in conference proceedings; and (4) were written in English, Portuguese, Spanish, or French. Articles were excluded if the study was conducted with animals or assessed ARSs with CORSA only at one specific moment in time. Book chapters, review papers, abstracts of communications or meetings, letters to the editor, commentaries to articles, unpublished work, and study protocols were not considered suitable and, therefore, were also excluded from this review.

\section{Study Selection}

First, duplicate articles were removed. Then, the title, abstract, and key words were analyzed to assess the type and relevance of the publication for the scope of the review. Finally, the full text of potentially relevant articles was independently screened for content by the 3 reviewers to decide on its inclusion in the review. Reviewers resolved disagreements by consensus.

\section{Data Extraction}

Data from the included articles were extracted in the following structured table format: first author's last name, year of publication, type of study, participants, intervention, data collection protocol, recording device, data analyses, ARS outcome, and findings.

\section{Quality Assessment}

The quality of the included studies was assessed with the checklist created by Downs and Black. ${ }^{14}$ The checklist provides a list of 27 questions to measure study quality, split into the following 5 sections: reporting (10 items); external validity ( 3 items); internal validity — bias ( 7 items); internal validity — confounding ( 7 items); and power (one item). Similar to previous systematic reviews, ${ }^{15,16}$ the scoring for question 27 , dealing with statistical power, was simplified to a choice of awarding either 1 point or 0 points, depending on whether there was sufficient power to detect a clinically important effect. Downs and Black ${ }^{14}$ score ranges were grouped into the following 4 quality levels: excellent (26-28), good (20-25), fair (15-19) and poor $(\leq 14) .{ }^{15,16}$ The risk of bias assessment was carried out by 2 independent reviewers. Disagreements between reviewers were resolved by reaching a consensus through discussion.

\section{Data Analysis}

To determine the consistency of the quality assessment performed by the 2 reviewers, an inter-rater agreement analysis using the Cohen's kappa was performed. The value of Cohen's kappa ranges from 0 to 1 and can be catego- 
rized as slight $(0.0-0.20)$, fair $(0.21-0.40)$, moderate $(0.41-$ $0.60)$, substantial (0.61-0.80), or almost perfect $(\geq 0.81)$ agreement. ${ }^{17}$ This statistical analysis was performed using PASW Statistics (version 18.0, SPSS, Chicago, Illinois). When quantitative pooling was appropriate, the effect sizes (ESs) together with the 95\% CIs were computed for the outcomes of interest. The ESs were interpreted as low (0.20), medium (0.50), and high (0.80) effect magnitudes. ${ }^{18}$ These quantitative data analyses were performed using the meta-analysis software Comprehensive Meta-Analysis, version 2 (Biostat, Englewood, New Jersey). ${ }^{19}$

\section{Results}

\section{Study Selection}

The database search identified 1,224 records. After the removal of duplicates, 900 records were screened for relevant content. During the title, abstract, and key word screening, 876 articles were excluded. The full text of the 24 potentially relevant articles was assessed, and 18 articles were excluded for the following reasons: ARS detection was performed with standard auscultation $(n=2)$ or manual annotation $(n=4)$; ARS automatic detection occurred at only one specific time point to validate algorithms $(n=5)$; data was not provided on ARSs $(n=3)$; and an intervention was absent $(n=4)$. Six original articles were included in the review. The search for relevant articles within the reference list of the selected articles retrieved 6 studies, which were also included (Fig. 1).

\section{Quality Assessment}

The articles included in this review scored 10 to 21 on the Downs and Black ${ }^{14}$ checklist, with a mean of $14.42 \pm 0.93$ (Table 1). Results of the risk of bias assessment indicated that 7 studies (64\%) had poor quality, 4 had fair quality, and one had good quality. Studies scored particularly poorly on the following items: description of confounders, sample representativeness, patient blinding, outcome assessor blinding, recruitment, randomization, adjustment for confounding factors in the analysis, and power to detect outcomes that are clinically important. The agreement between 2 reviewers was almost perfect (kappa $=0.825,95 \% \mathrm{CI}=0.758-0.885, P=.001)$.

\section{Study Characteristics}

The majority of the included studies were quasi-experimental, ,50-25 3 studies were observational, ${ }^{26-28}$ and 2 studies were randomized controlled trials (Table 2).29,30 Ten studies recruited patients receiving specialized care, and 2 studies recruited patients during hospital admission. ${ }^{24,27} \mathrm{~A}$ total of 275 subjects ( $n=126 ; 45.8 \%$ male) participated in

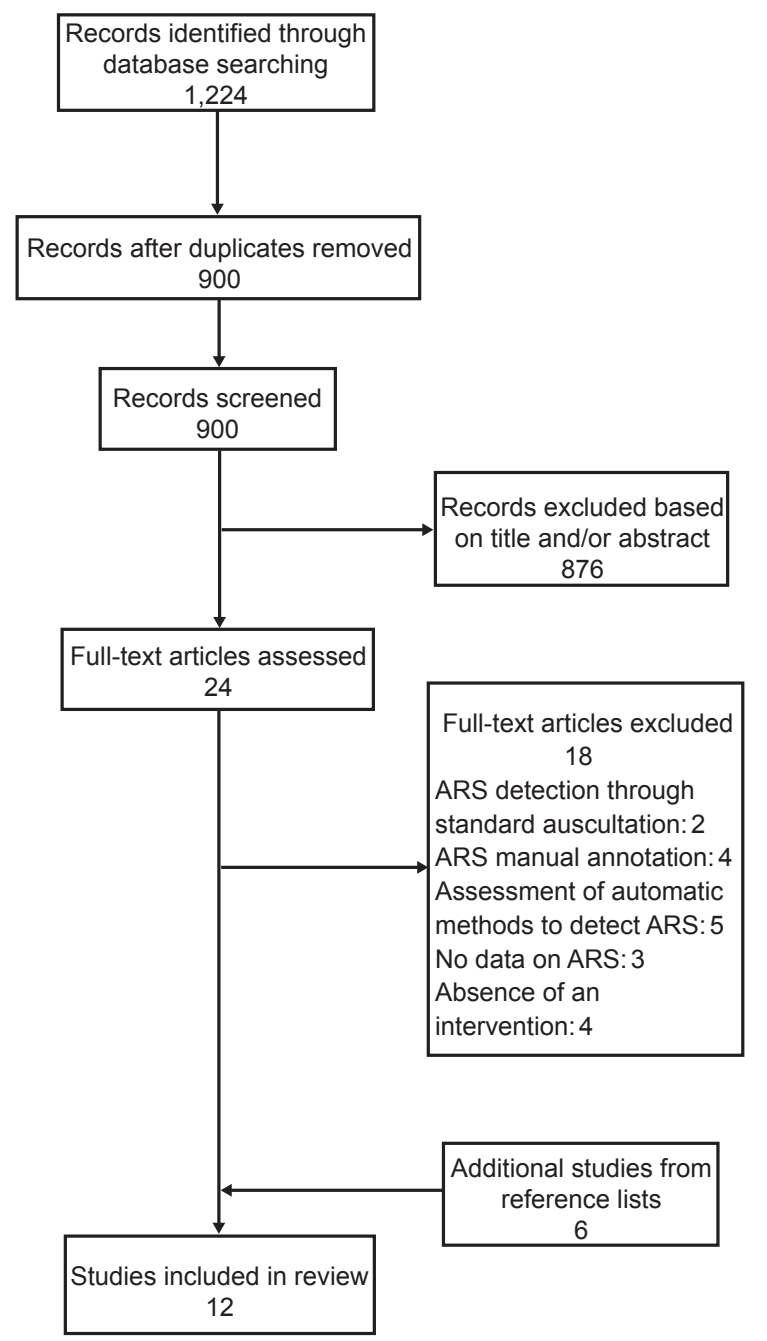

Fig. 1. PRISMA flowchart of the included studies. ARS = adventitious respiratory sounds.

the included studies; 47 were healthy subjects and 208 had respiratory conditions (asthma, $n=84$; pneumonia, $n=11$; cystic fibrosis, $n=23$; COPD, $n=6$; prolonged cough, $n=28$; bronchiolitis, $n=27$; bronchiectasis, $n=23$; and lower respiratory tract infection [LRTI], $n=26$ ). Eight studies were conducted with adults $(n=167 ; 60.7 \%$; age range $21-73$ years $)^{5,21,24-29}$ and 4 with children $(n=108$ [39.3\%]; age range 4 months-18 y).20,22,23,30

The interventions in most studies consisted of pharmacotherapy ${ }^{20-30}$; only 2 studies combined pharmacotherapy with respiratory physical therapy. ${ }^{5,29}$ The respiratory physical therapy consisted mainly of an active cycle of breathing techniques, $, 5,29$ but also breathing retraining techniques (incentive spirometry, thoracic mobility, expansion and flexibility exercises, and aerobic training). ${ }^{29}$ In almost all studies, the respiratory sounds were recorded in more than one chest location; however, in 3 studies recordings were performed exclusively in the trachea..125,26 Only the 3 
Table 1. Quality Assessment Score for Selected Studies Based on the Downs and Black ${ }^{14}$ Checklist

\begin{tabular}{|c|c|c|c|c|c|c|}
\hline Study & $\begin{array}{l}\text { Reporting } \\
(\mathrm{MS}=11)\end{array}$ & $\begin{array}{l}\text { External Validity } \\
\quad(\mathrm{MS}=3)\end{array}$ & $\begin{array}{l}\text { Internal Validity-Bias } \\
\quad(\mathrm{MS}=7)\end{array}$ & $\begin{array}{l}\text { Internal Validity-Confounding } \\
\quad(\mathrm{MS}=6)\end{array}$ & $\begin{array}{c}\text { Power } \\
(\mathrm{MS}=1)\end{array}$ & $\begin{array}{l}\text { Total } \\
\text { Score }\end{array}$ \\
\hline Baughman and Loudon ${ }^{24}$ & 5 & 0 & 6 & 1 & 0 & 12 \\
\hline Piirila $^{27}$ & 7 & 1 & 3 & 2 & 0 & 13 \\
\hline Sanchez et al ${ }^{20}$ & 6 & 1 & 4 & 0 & 0 & 11 \\
\hline Fiz et $\mathrm{al}^{21}$ & 7 & 0 & 5 & 0 & 0 & 12 \\
\hline Bentur et $\mathrm{al}^{23}$ & 8 & 0 & 4 & 1 & 0 & 13 \\
\hline Bentur et $\mathrm{al}^{22}$ & 8 & 2 & 5 & 2 & 1 & 18 \\
\hline Cortes et $\mathrm{al}^{26}$ & 6 & 1 & 1 & 2 & 0 & 10 \\
\hline Fiz et $\mathrm{al}^{25}$ & 8 & 0 & 5 & 3 & 0 & 16 \\
\hline Beck et $\mathrm{al}^{30}$ & 8 & 1 & 7 & 5 & 0 & 21 \\
\hline Marques et $\mathrm{a}^{5}$ & 8 & 1 & 5 & 2 & 0 & 16 \\
\hline Oliveira et $\mathrm{al}^{28}$ & 7 & 1 & 4 & 2 & 0 & 14 \\
\hline Dinis et $\mathrm{al}^{29}$ & 8 & 1 & 5 & 3 & 0 & 17 \\
\hline
\end{tabular}

more recent studies $5,28,29$ acquired the respiratory sounds following the CORSA guidelines for short-term acquisition. The recording devices used varied among studies, as follows: microphones ${ }^{21,24,25,27,29}$; piezoelectric sensors $20,22,23,26,30$; and electronic stethoscopes. 5,28

Algorithms based on fast Fourier transformation were the most used to automatically detect ARSs. Two studies $^{28,29}$ used an algorithm based on short-time Fourier transformation, and one study ${ }^{25}$ used a modification of the algorithm proposed by Shabtai-Musih et $\mathrm{al}^{31}$ and HomsCorbera et al. ${ }^{32}$ A total of 9 studies analyzed WHs (3 studies were conducted in children), 2 studies analyzed CRs, ${ }^{5,27}$ and one study analyzed both WHs and CRs in children. ${ }^{30}$ Two studies detected breathing cycles automatically; one study ${ }^{5}$ used an analogous method reported by Qiu et al, ${ }^{33}$ and the other study ${ }^{30}$ used the algorithm of Huq and Moussavi. ${ }^{34}$ Only 3 studies ${ }^{26,27,29}$ considered the breathing phases (inspiration and expiration) in the analysis of the ARSs.

\section{Synthesis of the Results}

\section{WHs}

Presence. The presence of WHs was used to identify a bronchial response during bronchial provocation tests in 2 studies conducted with children. ${ }^{20,22}$ Sanchez et al ${ }^{20}$ used concentrations of methacholine, and found that WHs had $50 \%$ sensitivity and $100 \%$ specificity to detect bronchial hyper-reactivity. Bentur et $\mathrm{al}^{22}$ observed that WHs were detected at a mean adenosine concentration of $15.6 \mathrm{mg} /$ $\mathrm{mL}$. Both studies verified that WHs were feasible to assess bronchial reactivity.

Number. Two studies investigated the number of WHs in adults. Oliveira et $\mathrm{al}^{28}$ found a significant increase in the number of WHs after 3 weeks of standard medical treatment (6 vs 14.8, $P=.03$; $\mathrm{ES}=4.38$ ) in patients with LRTI. A similar result, but not significant, was found in stable subjects with asthma after terbutaline inhalation (ES = 0.34); however, in healthy subjects and nonstable subjects with asthma a nonsignificant decrease was observed $\left(\mathrm{ES}=-0.10\right.$ and -0.012 , respectively). ${ }^{25}$

Frequency. The frequency of WHs was investigated in 4 studies conducted in adults. ${ }^{21,25,28,29}$ After terbutaline inhalation, the frequency of WHs significantly decreased in patients with asthma (ES $=-0.15)$ and COPD (ES $=-0.21)$, and in healthy subjects $(\mathrm{ES}=-0.28) .{ }^{21} \mathrm{Sim}-$ ilar, although nonsignificant, results were found with the same intervention in healthy subjects $(\mathrm{ES}=-0.18)$, and nonstable subjects with asthma $\left(\mathrm{ES}=\right.$ non-stable 0.24). ${ }^{25}$ In stable subjects with asthma $(\mathrm{ES}=0.01)^{25}$ and LRTI $(\mathrm{ES}=-0.06),{ }^{28}$ the frequency remained approximately the same. Dinis et al ${ }^{29}$ investigated the effect of respiratory physical therapy in subjects with LRTI, and observed a nonsignificant increase in the frequency of inspiratory and expiratory WHs in both of the experimental $(\mathrm{ES}=0.73$ and $\mathrm{ES}=0.04$, respectively) and control groups $(\mathrm{ES}=0.97$ and $\mathrm{ES}=0.97$, respectively).

Occupation Rate. This parameter, which is the proportion of the respiratory cycle occupied by WHs, was explored in studies conducted with children and adults. In 3 studies, ${ }^{24,25,30}$ the WH occupation rate (WH\%) was used to analyze the effect of pharmacotherapy. A nonsignificant reduction in $\mathrm{WH} \%$ during the night in the group of subjects to whom long-acting sympathomimetic agent had been administered (ES $=-1.9$ ) was found; whereas, in the placebo group a significant increase was observed $(\mathrm{ES}=1.15) .{ }^{24}$ In a study ${ }^{30}$ conducted with infant viral 


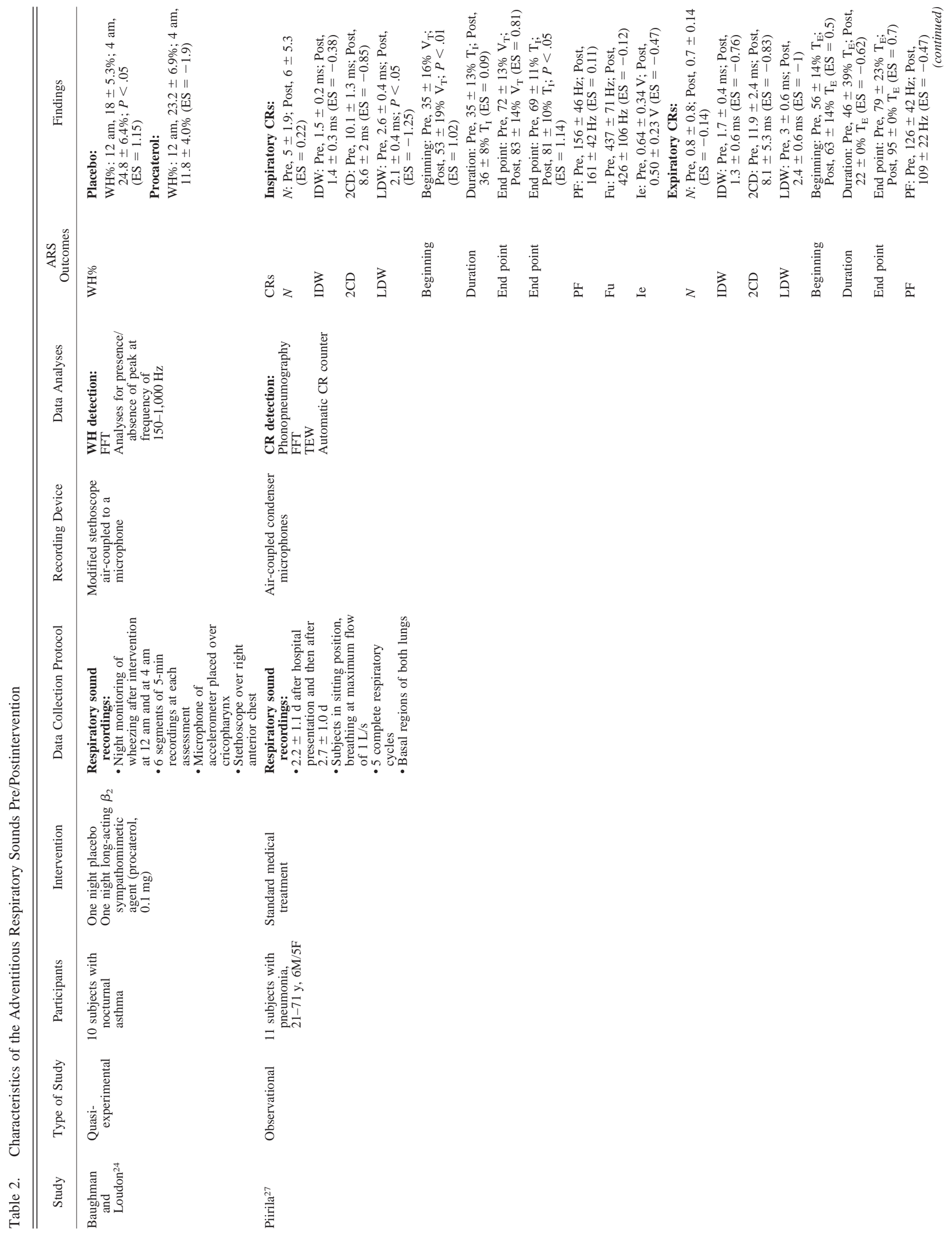




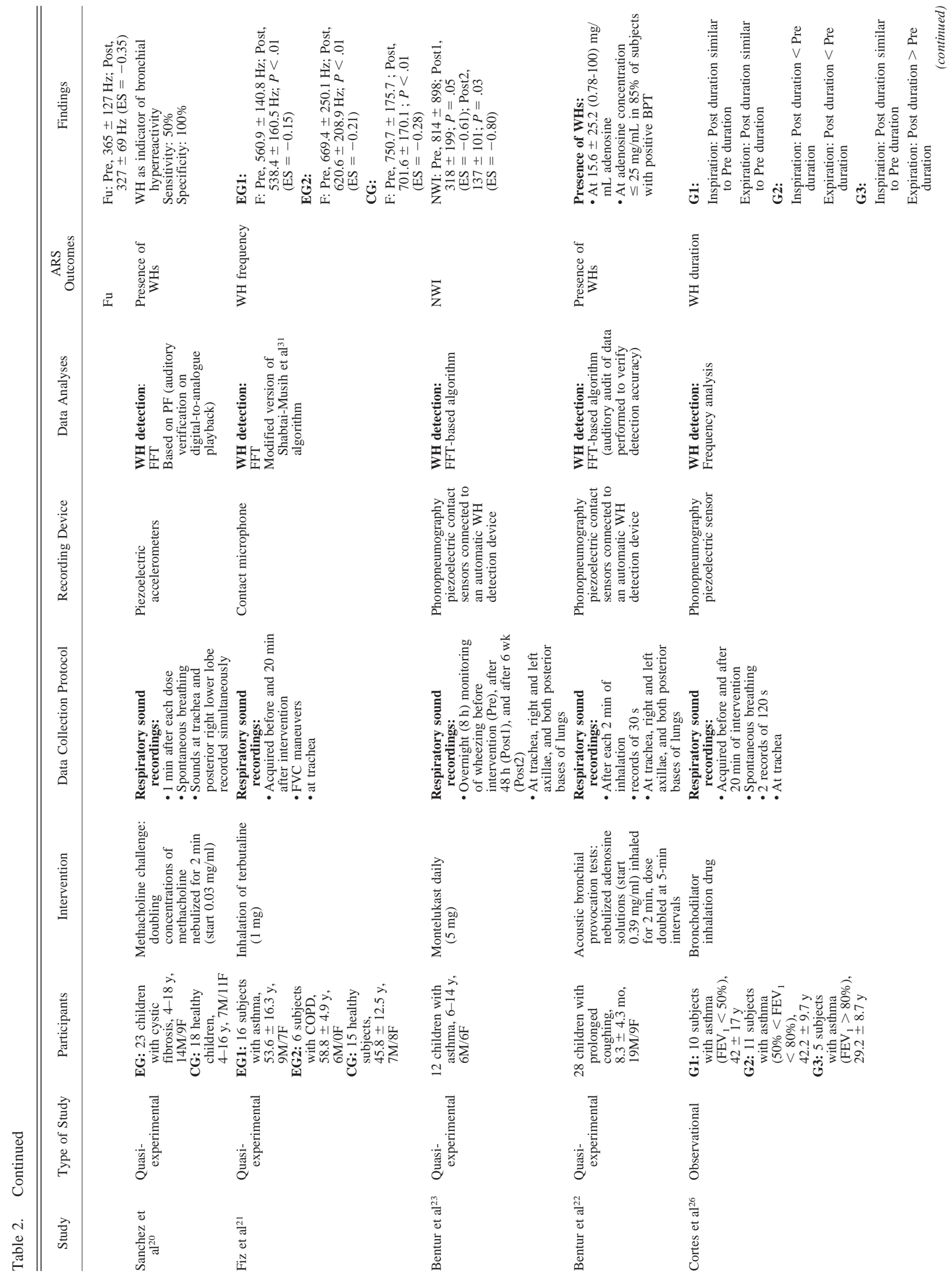




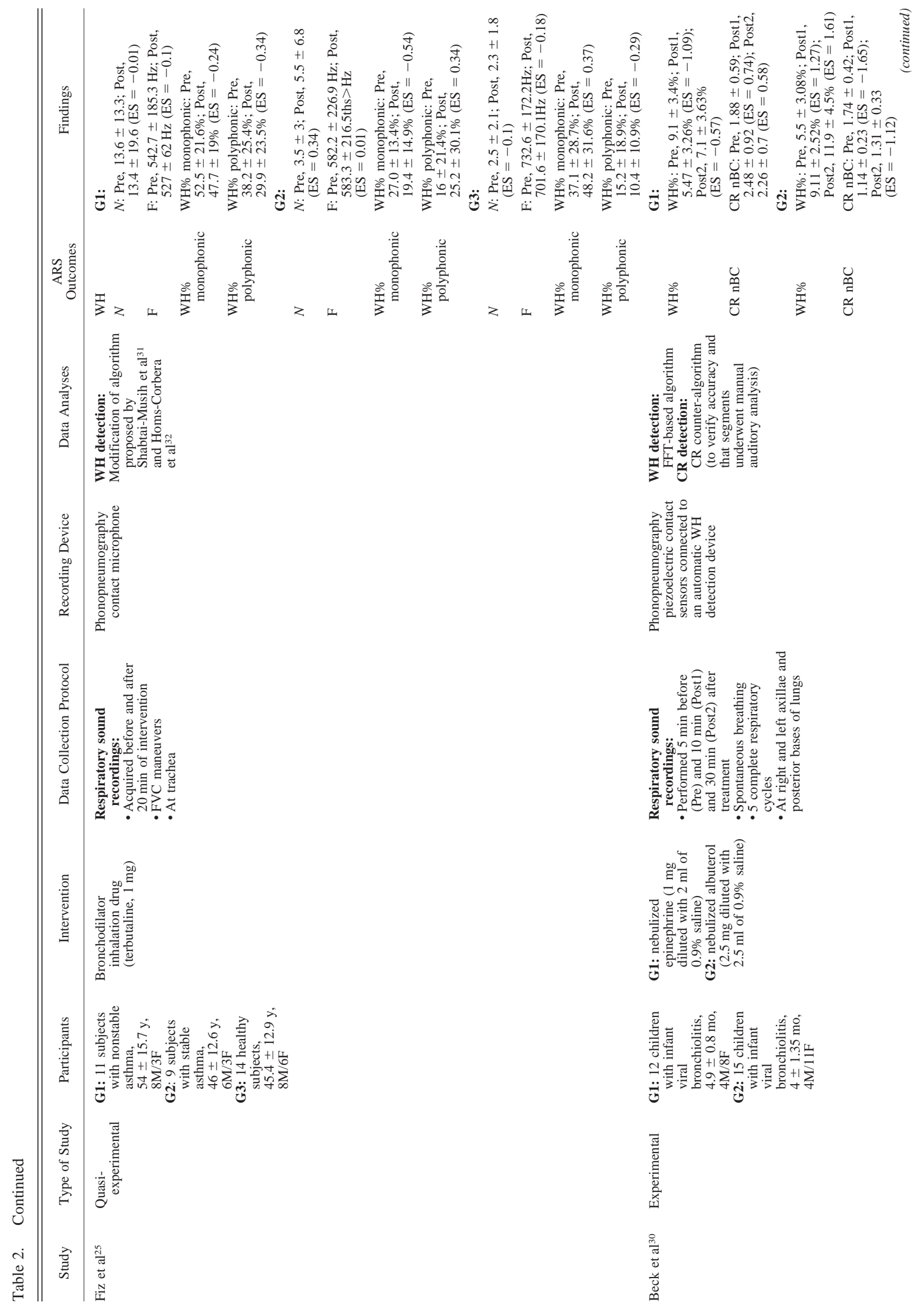




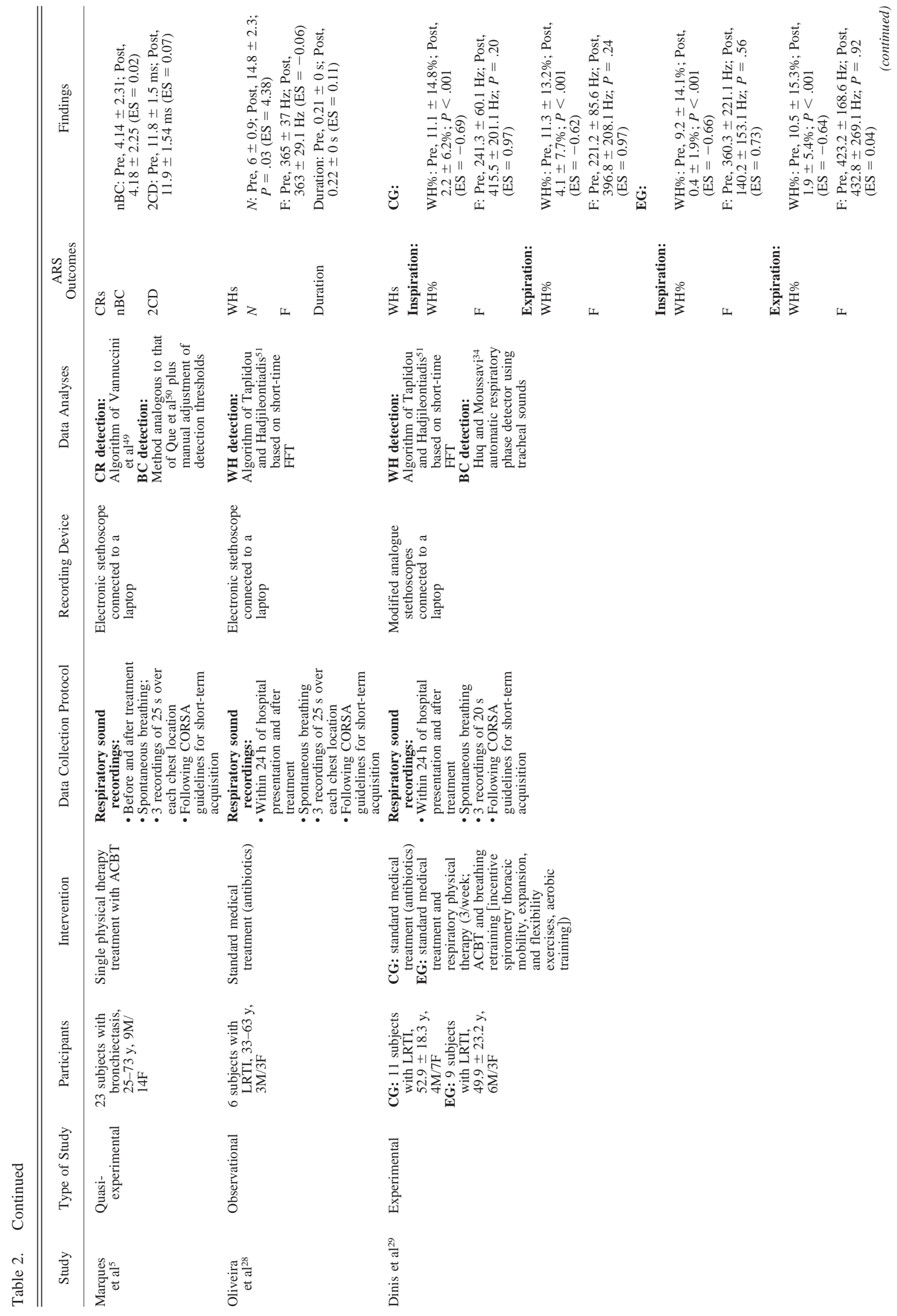




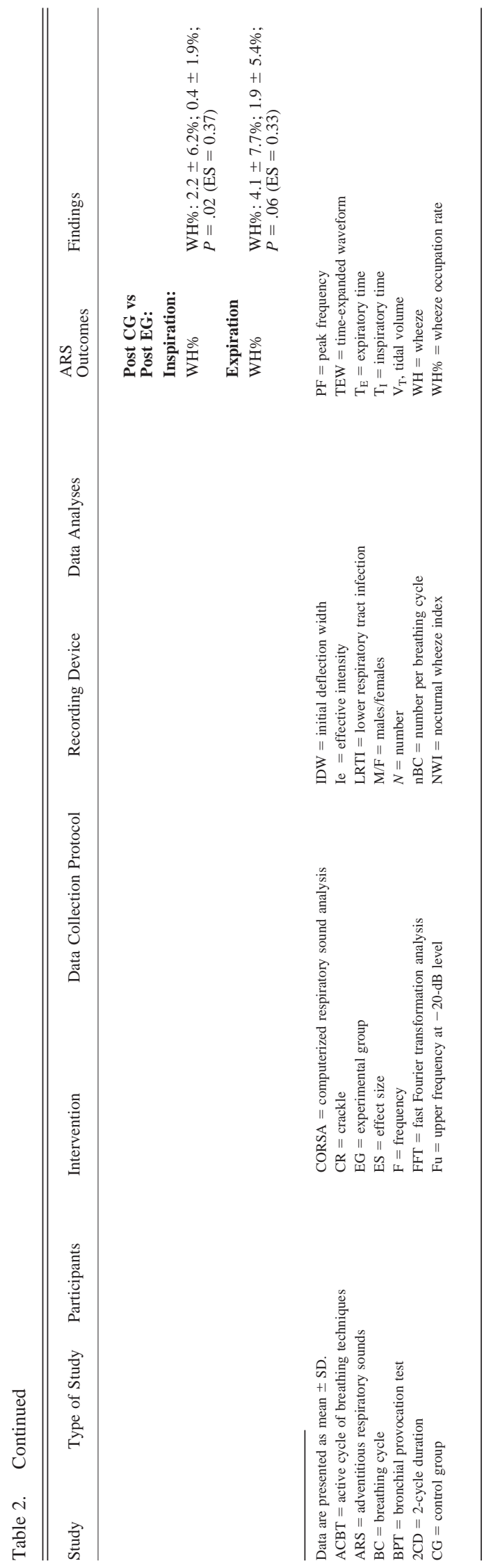

bronchiolitis, WH\% also decreased 10 min after the administration of epinephrine (ES $=-1.09$ ); however, WH\% increased in the group of children to albuterol had been administered $(\mathrm{ES}=1.27)$. Nevertheless, no significant differences were found between or within groups. When exploring monophonic and polyphonic $\mathrm{WH} \%$, significant change was also not found; however, low ESs in nonstable and healthy subjects, and medium ESs in stable subjects with asthma (ES $=-0.54)$ were found. ${ }^{25}$ A significant decrease in inspiratory and expiratory $\mathrm{WH} \%$ was found after 3 weeks of pharmacotherapy plus respiratory physical therapy $(\mathrm{ES}=-0.66$ [inspiratory]; $\mathrm{ES}=-0.64$ [expiratory]) or pharmacotherapy alone $(\mathrm{ES}=-0.69$ [inspiratory]; ES $=-0.62$ [expiratory]). ${ }^{29} \mathrm{~A}$ similar result was found for the nocturnal WH index, calculated from the WH\% (after $2 \mathrm{~d}$ [ES $=-0.61]$ and after 6 weeks [ES $=-0.80]$ ), when monitoring respiratory sounds overnight to assess the effects of montelukast in patients with nocturnal asthma. ${ }^{23}$

Duration. Two studies ${ }^{26,28}$ explored this variable when assessing the impacts of pharmacotherapy with adult subjects. In both studies, WH duration remained approximately the same before and after intervention. Only in subjects with moderate and severe obstruction, changes in the duration of WHs after medication were observed. ${ }^{26}$

\section{CRs}

Number. Three studies analyzed the number of CRs before and after intervention, and no significant differences were found. In 2 studies, this variable remained approximately the same, with ESs ranging from 0.02 to $0.22 .5,27$ In the study by Beck et al, ${ }^{30}$ the number of CRs increased $(E S=0.58)$ with albuterol therapy and decreased with epinephrine therapy $(\mathrm{ES}=-1.65)$.

Frequency. The CR frequency was analyzed only in one study. ${ }^{27}$ The peak frequency increased during inspiration $(\mathrm{ES}=0.11)$ and decreased during expiration (ES $=-0.47)$, whereas the upper frequency at the $-20-\mathrm{dB}$ level decreased in both inspiration $(\mathrm{ES}=-0.12)$ and expiration $(E S=-0.35)$. No significant differences were found.

2-Cycle Duration, Largest Deflection Width, and Initial Deflection Width. Two studies analyzed the 2-cycle duration (2CD) variable; Marques et $\mathrm{al}^{5}$ did not show any change from before to after intervention $(\mathrm{ES}=0.07)$, and Piirila ${ }^{27}$ showed a nonsignificant reduction postintervention both in the inspiratory ( $\mathrm{ES}=-0.85$ ) and expiratory $(\mathrm{ES}=-0.83)$ phases. In the study by Piirila, ${ }^{27}$ both the largest deflection width (LDW) and the initial deflection 
width (IDW) of inspiratory CRs (ES $=-1.25$ and -0.38 , respectively) and expiratory CRs (ES $=-1$ and -0.76$)$ were shorter after the intervention.

Timing. Only Piirila ${ }^{27}$ explored the timing parameters of the CRs related to inspiratory tidal volume, and inspiratory and expiratory phases. These parameters were significantly different postintervention (ESs $0.5-1.14$ ).

Due to the heterogeneity of the outcome measures used across studies, a meta-analysis was not possible to compute.

\section{Discussion}

The main finding of this systematic review was that ARSs detected and characterized by CORSA show potential to be used as outcome measures in children and adults, as specific variables of each ARS presented high ESs. However, the most appropriate variable or variables are yet to be explored.

Most studies (11 of 12) explored the presence and characteristics of WHs before/after an intervention. The WH\% seemed to be the most promising parameter to be used as an outcome measure in children and adults, with medium to high ESs varying from 0.62 to $1.9 .^{24,29}$ A strong association between the proportion of the respiratory cycle occupied by WHs and the degree of bronchial obstruction has been widely demonstrated. ${ }^{21-23,35}$ This WH parameter, even when identified with standard auscultation, has shown to be sensitive to assess the effectiveness of respiratory interventions in children. ${ }^{36,37}$ The WH complexity may also be a variable of interest as the presence of polyphonic WHs indicates a more serious obstruction than monophonic $\mathrm{WHs}^{25}$; however, this was explored in only one study (ESs $0.24-0.54) .{ }^{25} \mathrm{WH}$ monitoring has been found to provide more information on the changes in airway obstruction than measurements of pulmonary function, ${ }^{24}$ such as the $\mathrm{FEV}_{1}$ percent predicted in people with asthma. ${ }^{26}$ Thus, WHs and their variables seem to be a promising objective outcome measure for all populations with a special emphasis on noncollaborative populations such as children, people with dementia, and people in the ICU. However, it should be noticed that WHs occur only when there is a flow limitation (but flow limitation is not necessarily accompanied by WHs) that reaches a critical value, which is called "flutter velocity." 38 Thus, when there is not enough flow to generate WHs, WH parameters will not be useful despite the presence of the respiratory problem. The complementary information provided by CRs is, therefore, crucial.

CRs are assumed to be caused by the sudden opening of abnormally closed airways, ${ }^{39-42}$ and their parameters provide essential information about the function and structure of the tracheobronchial tree ${ }^{41}$ (eg, CR recording during mechanical ventilation has been considered a simple method to monitor lung recruitment/derecruitment). ${ }^{43} \mathrm{How}-$ ever, CR variables have been explored as outcome measures in only 3 studies. ${ }^{5,27,30}$ From the limited evidence available, LDW seemed to be the most valuable parameter to be used as an outcome measure due to its high ESs (1 and 1.25). ${ }^{27}$ Hoevers and Loudon ${ }^{44}$ had already found that LDW seemed to be a better measure than IDW or $2 \mathrm{CD}$ when differentiating between coarse and fine CRs. However, LDW was also the variable less explored among studies. Conflicting information was found for the number of CRs and 2CD. The number of CRs had low ESs reported in studies by Piirila ${ }^{27}$ (0.14 and 0.22) and Marques et $\mathrm{al}^{5}(0.02)$, and medium/high ESs in the study by Beck

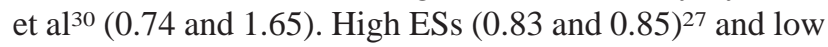
ESs $(0.07)^{5}$ were also found for the variable $2 \mathrm{CD}$. The timing of CRs (ESs 0.5-1.14) was also shown to be sensitive to the clinical course of pneumonia ${ }^{27}$ and has been described as a sensitive parameter with which to discriminate respiratory diseases. ${ }^{45}$ However, similarly to LDW, limited research has been conducted considering this parameter as an outcome measure. At this point in time, it is difficult to provide any recommendations in which the $\mathrm{CR}$ variable is more adequate as an outcome measure to monitor respiratory interventions. These limited and conflicting data may be a result of the different respiratory sound acquisition sensors (which differ in their acoustic sensitivity to capture CR waveforms), filtering, and analysis methods used across studies. ${ }^{46,47}$ Since CRs show potential for diagnostic purposes but also as an outcome measure for respiratory treatments in different clinical/research contexts, these procedures need to be standardized. This will allow comparisons among different studies and improve the understanding of the mechanisms and acoustic characteristics of CRs.

The study of both main types of ARSs is essential to gather complementary information about the tracheobronchial tree. This information may help health professionals to conduct more accurate diagnoses and enhance their understanding about the respiratory system responses to treatments. However, only one study ${ }^{30}$ analyzed both types of ARSs as an outcome measure in a study conducted with children. Thus, the study of computerized ARSs is an exciting area where much research is needed to develop knowledge for the diagnosis of conditions and the monitoring of patients, but also for use as a noninvasive, objective, and reliable outcome measure for treatment.

The level of evidence that can be drawn at this moment in time from this systematic review is considerably weak due to the (1) small sample sizes; (2) distinct respiratory therapies and doses implemented, and (3) different ARS variables used in the included studies. The large variety of acquisition methods used is an issue added to the list of difficulties when comparing results across studies. A 


\section{ARSs as Outcome Measures for Respiratory Therapy}

BIOMED 1 Concerted Action project entitled CORSA, funded by the European Community, developed guidelines for research and clinical practice in the field of respiratory sound acquisition and analysis., ${ }^{4,9,48}$ The CORSA project group produced guidelines on the definitions of medical/ technical terms used in pulmonary acoustics; environmental conditions; patient management procedures; acquisition, pre-processing, digitization and analysis of respiratory sounds; and also on publishing the results of research. ${ }^{4,9,48}$ These international guidelines have been available since 2000; however, from the 9 studies conducted after this year, only 3 followed the acquisition procedures recommended by CORSA. Regarding the analysis methods, algorithms based on fast Fourier transformation were the most used, which is in accordance with the CORSA recommendations. Future research, with improved study designs and larger samples, both of children and adult populations, and following the CORSA guidelines, should be conducted to explore the ARS response to respiratory therapies. This will facilitate the comparison of results from different studies, promote research into the development of standardized respiratory sound acquisition equipment and analysis, and, finally, enhance the understanding of computerized ARSs as well as their use as an outcome measure.

\section{Conclusion}

Specific variables of each ARS detected and characterized with CORSA showed high ESs, and thus may have potential to be an objective, reliable, and noninvasive outcome measure for respiratory therapy in children and adults. Further research exploring the ARS response to different respiratory therapies is needed to enhance the understanding of computerized ARSs and their clinical use, not only for purposes of diagnosis but also for monitoring patients and treatments.

\section{REFERENCES}

1. World Health Organization. The World Health Report 2008: primary health care-now more than ever. World Health Organization, Geneva, Switzerland 2008.

2. Hayes D Jr, Kraman SS. The physiologic basis of spirometry. Respir Care 2009;54(12):1717-1726.

3. Marques A, Bruton A, Barney A. Clinically useful outcome measures for physiotherapy airway clearance techniques: a review. Phys Ther Rev 2006;11(4):299-307.

4. Sovijarvi ARA, Dalmasso F, Vanderschoot J, Malmberg LP, Righini G, Stoneman SAT. Definition of terms for application of respiratory sounds. Eur Respir Rev 2000;10(77):597-610.

5. Marques A, Bruton A, Barney A, Hall A. Are crackles an appropriate outcome measure for airway clearance therapy? Respir Care 2012; 57(9):1468-1475.

6. Vyshedskiy A, Murphy R. Crackle pitch rises progressively during inspiration in pneumonia, CHF, and IPF patients. Pulm Med 2012; 2012:240160.
7. Gavriely N, Nissan M, Cugell DW, Rubin AHE. Respiratory health screening using pulmonary function tests and lung sound analysis. Eur Respir J 1994;7(1):35-42.

8. Sovijarvi ARA, Malmberg LP, Charbonneau G, Vanderschoot J, Dalmasso F, Sacco C, et al. Characteristics of breath sounds and adventitious respiratory sounds. Eur Respir Rev 2000;10(77):591596.

9. Sovijarvi ARA, Vanderschoot J, Earis JE. Standardization of computerized respiratory sound analysis. Eur Respir Rev 2000;10(77): 585 .

10. Pasterkamp H. Accoustic markers of airway responses during inhalation challenge in children. Pediatr Pulmonol Suppl 2004;26:175176.

11. Murphy RLH. In defense of the stethoscope. Respir Care 2008; 53(3):355-369.

12. Gurung A, Scrafford C, Tielsch J, Levine O, Checkley W. Computerized lung sound analysis as diagnostic aid for the detection of abnormal lung sounds: a systematic review and meta-analysis. Respir Med 2012;105(9):1396-1403.

13. Moher D, Liberati A, Tetzlaff J, Altman DG, PRISMA Group. Preferred reporting items for systematic reviews and meta-analyses: the PRISMA statement. Ann Intern Med 2009;151(4):264-269.

14. Downs SH, Black N. The feasibility of creating a checklist for the assessment of the methodological quality both of randomised and non-randomised studies of health care interventions. J Epidemiol Community Health 1998;52(6):377-384.

15. Chudyk AM, Jutai JW, Petrella RJ, Speechley M. Systematic review of hip fracture rehabilitation practices in the elderly. Arch Phys Med Rehabil 2009;90(2):246-262.

16. Samoocha D, Bruinvels DJ, Elbers NA, Anema JR, van der Beek AJ. Effectiveness of web-based interventions on patient empowerment: a systematic review and meta-analysis. J Med Internet Res 2010;12(2): e23.

17. Landis JR, Koch GG. The measurement of observer agreement for categorical data. Biometrics 1977;33(1):159-174.

18. Cohen J. Statistical power analysis for the behavioral sciences. Hillsdale, NJ:Lawrence Earlbaum;1988.

19. Borenstein M, Hedges L, Higgins J, Rothstein H. Comprehensive meta-analysis 2.0. Englewood, NJ:Biostat;2005.

20. Sanchez I, Powell RE, Pasterkamp H. Wheezing and airflow obstruction during methacholine challenge in children with cystic fibrosis and in normal children. Am Rev Respir Dis 1993;147(3):705709.

21. Fiz JA, Jane R, Homs A, Izquierdo J, Garcia MA, Morera J. Detection of wheezing during maximal forced exhalation in patients with obstructed airways. Chest 2002;122(1):186-191.

22. Bentur L, Beck R, Berkowitz D, Hasanin J, Berger I, Elias N, et al. Adenosine bronchial provocation with computerized wheeze detection in young infants with prolonged cough: correlation with longterm follow-up. Chest 2004;126(4):1060-1065.

23. Bentur L, Beck R, Shinawi M, Naveh T, Gavriely N. Wheeze monitoring in children for assessment of nocturnal asthma and response to therapy. Eur Respir J 2003;21(4):621-626.

24. Baughman RP, Loudon RG. The utility of the estimated Tw/Ttot in evaluating a long acting sympathomimetic agent used for nocturnal asthma. Annu Rev Chronopharmacol 1988;5:157-160.

25. Fiz JA, Jane R, Izquierdo J, Homs A, Garcia MA, Gomez R, et al. Analysis of forced wheezes in asthma patients. Respiration 2006; 73(1):55-60.

26. Cortes S, Jane R, Fiz JA, Morera J. Monitoring of wheeze duration during spontaneous respiration in asthmatic patients. Conf Proc IEEE Eng Med Biol Soc 2005;6:6141-6144.

27. Piirila P. Changes in crackle characteristics during the clinical course of pneumonia. Chest 1992;102(1):176-183. 


\section{ARSs as Outcome Measures for Respiratory Therapy}

28. Oliveira A, Pinho C, Dinis J, Oliveira D, Marques A. Automatic wheeze detection and lung function evaluation: a preliminary study. Paper presented at International Conference on Health Informatics, Biomedical Engineering Systems and Technologies 6th International Joint Conference, BIOSTEC 2013, Barcelona, 2013.

29. Dinis J, Oliveira A, Pinho C, Campos G, Rodrigues J, Marques A. Automatic wheeze and respiratory phase detectors to evaluate respiratory physiotherapy in LRTI: a preliminary study. Paper presented at International Conference on Health Informatics, Biomedical Engineering Systems and Technologies 6th International Joint Conference, BIOSTEC 2013, Barcelona, 2013.

30. Beck R, Elias N, Shoval S, Tov N, Talmon G, Godfrey S, et al. Computerized acoustic assessment of treatment efficacy of nebulized epinephrine and albuterol in RSV bronchiolitis. BMC Pediatr 2007; 7(22):1-6.

31. Shabtai-Musih Y, Grotberg JB, Gavriely N. Spectral content of forced expiratory wheezes during air, He, and SF6 breathing in normal humans J Appl Physiol 1992;72(2):629-635.

32. Homs-Corbera A, Fiz JA, Jane R. Time-frequency detection and analysis of wheezes during forced exhalation. IEEE Trans Biomed Eng 2004;51(1):182-186.

33. Qiu Y, Whittaker AR, Lucas M, Anderson K. Automatic wheeze detection based on auditory modelling. Proc Inst Mech Eng H 2005; 219(3):219-227.

34. Huq S, Moussavi Z. Automatic breath phase detection using only tracheal sounds. Conf Proc IEEE Eng Med Biol Soc 2010;2010:272275.

35. Baughman RP, Loudon RG. Quantitation of wheezing in acute asthma. Chest 1984;86(5):718-722.

36. Postiaux G, Louis J, Labasse HC, Gerroldt J, Kotik AC, Lemuhot A, et al. Evaluation of an alternative chest physiotherapy method in infants with respiratory syncytial virus bronchiolitis. Respir Care 2011;56(7):989-994.

37. Gomes EL, Postiaux G, Medeiros DR, Monteiro KK, Sampaio LM, Costa D. Chest physical therapy is effective in reducing the clinical score in bronchiolitis: randomized controlled trial. Rev Bras Fisioter 2012;16(3):241-247.

38. Grotberg JB, Gavriely N. Flutter in collapsible tubes: a theoretical model of wheezes. J Appl Physiol 1989;66(5):2262-2273.
39. Pasterkamp H, Kraman SS, Wodicka GR. Respiratory sounds - advances beyond the stethoscope. Am J Respir Crit Care Med 1997; 156(3):974-987.

40. Alencar AM, Hantos Z, Petak F, Tolnai J, Asztalos T, Zapperi S, et al. Scaling behavior in crackle sound during lung inflation. Phys Rev E Stat Phys Plasmas Fluids Relat Interdiscip Topics 1999;60(4): 4659-4663.

41. Alencar AM, Majumdar A, Hantos Z, Buldyrev SV, Eugene Stanley $\mathrm{H}$, Suki B. Crackles and instabilities during lung inflation. Physica A 2005;357(1):18-26

42. Alencar AM, Buldyrev SV, Majumdar A, Stanley HE, Suki B. Avalanche dynamics of crackle sound in the lung. Phys Rev Lett 2001; 87(8):088101.

43. Peták F, Habre W, Babik B, Tolnai J, Hantos Z. Crackles-sound recording to monitor airway closure and recruitment in ventilated pigs. Eur Respir J 2006;27(4):808-816.

44. Hoevers J, Loudon RG. Measuring crackles. Chest 1990;98(5):12401243

45. Piirila P, Sovijarvi ARA. Crackles: recording, analysis and clinical significance. Eur Respir J 1995;8(12):2139-2148

46. Katila T, Piirila P, Kallio K, Paajanen E, Rosqvist T, Sovijarvi AR. Original waveform of lung sound crackles: a case study of the effect of high-pass filtration. J Appl Physiol 1991;71(6):2173-2177.

47. Kraman SS, Wodicka GR, Pressler GA, Pasterkamp H. Comparison of lung sound transducers using a bioacoustic transducer testing system. J Appl Physiol 2006;101(2):469-476.

48. Rossi M, Sovijarvi ARA, Piirila P, Vannuccini L, Dalmasso FVJ. Environmental and subject conditions and breathing manoeuvres for respiratory sound recordings. Eur Respir Rev 2000;10(77):611615 .

49. Vannuccini L, Rossi M, Pasquali G. A new method to detect crackles in respiratory sounds. Technol Health Care 1998;6(1):75-79.

50. Que CL, Kolmaga C, Durand LG, Kelly SM, Macklem PT. Phonospirometry for noninvasive measurement of ventilation: methodology and preliminary results. J Appl Physiol 2002;93(4):15151526.

51. Taplidou SA, Hadjileontiadis LJ. Wheeze detection based on timefrequency analysis of breath sounds. Comput Biol Med 2007;37(8): 1073-1083. 